\title{
Takeover Times on Scale-Free Topologies
}

\author{
Joshua L. Payne \\ Dept. of Computer Science \\ University of Vermont \\ Burlington, VT 05405 \\ 802-656-9116 \\ Joshua.Payne@uvm.edu
}

\author{
Margaret J. Eppstein \\ Dept. of Computer Science \\ University of Vermont \\ Burlington, VT 05405 \\ 802-656-1918 \\ Maggie.Eppstein@uvm.edu
}

\begin{abstract}
The topological properties of a network directly impact the flow of information through a system. In evolving populations, the topology of inter-individual interactions affects the rate of dissemination of advantageous genetic information and thus affects selective pressure. In this study, we investigate the selective pressures induced by several scale-free population structures using takeover time analysis. Previous results have shown that the selective pressures induced by scale-free interaction topologies are at least as strong as those induced by random and panmictic interaction topologies. In contrast, our results show that the selective pressures induced by scale-free interaction topologies are heavily influenced by their underlying topological properties, and can be tuned from a selective pressure close to that of a random or panmictic topology to a selective pressure that is weaker than that of a two-dimensional toroidal lattice with $3 \times 3$ rectangular neighborhoods of interactions. We also provide a detailed topological analysis of these population structures and discuss their influence on the observed dynamics in takeover times. We show that the expected takeover times observed on all population structures considered herein can be rapidly estimated using only a few readily computable metrics of the underlying topology, precluding the need to run expensive simulations or recursive probabilistic formulations.
\end{abstract}

\section{Categories and Subject Descriptors}

I.2.8 Artificial Intelligence [Problem Solving, Control Methods and Search]: Heuristic Methods

\section{General Terms}

Algorithms, Performance, Design, Experimentation

\section{Keywords}

Scale-free, Spatial Structure, Takeover Time, Selective Pressure

Permission to make digital or hard copies of all or part of this work for personal or classroom use is granted without fee provided that copies are not made or distributed for profit or commercial advantage and that copies bear this notice and the full citation on the first page. To copy otherwise, or republish, to post on servers or to redistribute to lists, requires prior specific permission and/or a fee.

GECCO '07, July 7-11, 2007, London, England, United Kingdom.

Copyright 2007 ACM 978-1-59593-697-4/07/007_..\$5.00.

\section{INTRODUCTION}

Network topology plays a large role in governing the flow of information through a system. For example, in evolving systems, different mating interaction topologies affect the rate of flow of genetic information and thus affect selective pressure. In classical quantitative genetics [8] and canonical evolutionary algorithms [12], mating interactions are typically assumed or allowed to be random. In such panmictic systems, deleterious alleles are rapidly eliminated and advantageous alleles spread quickly. In contrast, in the cellular evolutionary algorithm (cEA) [10][27][28], populations are structured on low-order regular graphs, such as one-dimensional and two-dimensional lattices, where mating events are restricted to occur within spatially localized, overlapping neighborhoods. In such lattice-structured topologies, the localization of recombination events mitigates selective pressure by slowing the dissemination of advantageous genetic information throughout the population [10]. Evolving natural biological populations also exhibit spatial structure in mating interactions that are often observed to be somewhere in between these two extremes of random and strictly local (e.g. [19]). Recent research has shown that many other types of interaction topologies of practical interest are also spatially structured. For example, "scale-free" topologies possessing a power law distribution of vertex connectivity have been shown to be pervasive in both natural and manmade systems [3], including the world wide web [1], protein-protein interactions [14], the internet [32], societal interactions [7][17], and semantic relationships between words in the English language [18]. In scale-free networks, the probability that a given node has $k$ connections follows a power law $\mathrm{P}(k)=k^{-\gamma}$, so while the majority of nodes have very few connections, a few nodes possess the majority of connections, acting as "hubs" in the network. Studying the flow of information through these networks can lead to insights into important processes such as network robustness [6][14] and spread of disease [23]. Thus, whether studying natural or artificial evolutionary or other interacting systems, it is important to understand the influence of the interconnection topology on the flow of information through the system.

One useful method for quantifying how the flow of information is controlled by a given population structure is through the analysis of takeover time [11]. Takeover time is defined as the expected number of generations until a population consists entirely of copies of the best individual, starting from only one copy of the best individual in the initial population. In evolutionary systems, this analysis removes the disruptive effects of recombination and mutation by letting selection act as the only evolutionary operator. 
Higher takeover times suggest lower selective pressure, and vice versa.

Takeover times in panmictic population structures have been thoroughly analyzed under a variety of selection mechanisms and have been shown to be quite rapid [11]. Takeover times in onedimensional and two-dimensional cEA's have also received a large amount of attention. Rudolph [27] analytically derived lower and upper bounds for one-dimensional non-toroidal lattices and exact solutions for one-dimensional toroidal lattices. Sarma and De Jong [28] analyzed two-dimensional toroidal lattices using several neighborhood sizes and shapes and showed that selection pressure is largely governed by the radius of the local mating neighborhood. Giacobini et al. [10] derived mathematical models of takeover times in one-dimensional and two-dimensional toroidal lattices with synchronous and asynchronous updating policies. The general result of these studies is that lattice population structures reduce selective pressure, relative to panmictic mating interactions, and can thus enhance the exploratory power of evolutionary search [10].

Giacobini et al. [9] extended their analysis of takeover time in regular one-dimensional and two-dimensional lattices to include non-regular topologies as well, including random graphs [20]. Their analytical approximations and empirical simulations showed that the selective pressure induced by random graph topologies is qualitatively similar to the selective pressure induced by panmictic population structures.

In their extension of takeover time analysis to non-regular topologies, Giacobini et al. [9] also studied one particular instance of a scale-free topology, generated using the algorithm proposed by Albert and Barabàsi ( $\mathrm{AB})$ [3]. Their empirical simulations of takeover times showed that the selective pressure induced by this particular AB scale-free topology was qualitatively similar to that induced by a random or panmictic population structure, when the initial copy of the best individual was equally likely to appear in any given node. They also showed that the takeover time in $\mathrm{AB}$ scale-free topologies can be dramatically reduced by strategically placing the initial copy of the best individual in a highly connected node.

While the $\mathrm{AB}$ algorithm produces topologies exhibiting the power-law distribution of vertex connectivity observed in many real-world scale-free topologies (e.g. [1][3]), it fails to capture some other important characteristics present in particular instances of real-world scale-free topologies, such as modularity and clustering. Accordingly, several algorithms were subsequently introduced [4][13][16][26] that better portrayed these particular characteristics.

The current work investigates takeover times induced by scalefree population structures generated using alternative scale-free graph generators. We show that the selective pressure induced by scale-free spatial topologies can be tuned from a selective pressure close to that of a random or panmictic topology to a selective pressure that is weaker than that of a two-dimensional toroidal lattice with $3 \times 3$ rectangular neighborhoods of interactions (i.e. Moore neighborhoods). We also provide a detailed structural analysis of these spatial topologies and discuss the influence of these structural characteristics on the observed dynamics in takeover times. This work represents a first step towards a unified empirical approach for quickly approximating network dynamics from readily computed characteristics of diverse topologies.

\section{METHODS}

\subsection{Population structure as a graph}

A graph, $G=(V, E)$, is defined as a nonempty finite set of vertices $(V)$ and a finite set of edges $(E)$ connecting these vertices. Representing the population structure of an evolutionary algorithm as a graph is relatively straightforward. Each individual in the population is represented by a vertex $i \in V$, so that $|V|=\mu$, where $\mu$ is the population size. An undirected edge $(i, j)$ is added to $E$ for each individual $j$ in the mating neighborhood of individual $i$, for all $i \in V$. One-dimensional (1D) and twodimensional (2D) toroidal lattices thus correspond to low-order regular graphs, wherein each vertex has the same degree (i.e. every individual has the same number of individuals in its mating neighborhood) and panmictic population structures correspond to complete graphs (i.e. fully connected regular graphs).

\subsection{Generating Scale-Free Graphs}

In this study, we employ three previously published scale-free graph-generating algorithms [3][13][26], which are described briefly in this section. Other scale-free graph generators have been proposed (e.g. [5][22][30]), but typically result in topologies consisting of one giant connected component and several small clusters of vertices that are isolated from the rest of the graph. Since individuals situated in the small fragmented clusters cannot communicate their genetic information to/from the rest of the population, such fragmentation unnecessarily reduces the overall population size. For this reason, we restrict our attention herein to graph generating algorithms that ensure connectivity.

\subsubsection{The Albert-Bararbàsi Algorithm}

The first algorithm presented to generate scale-free graphs was proposed by Albert and Barabàsi [3]. The algorithm is conceptually straightforward and hinges upon two key features: $(i)$ growth of the number of nodes in the network, and (ii) preferential attachment of new nodes to more highly connected nodes. The $\mathrm{AB}$ algorithm begins with an initial set of $m_{0}$ vertices that are not connected by any edges. The graph grows at each time step $t$ through the addition of a single new vertex, which attaches to $m\left(\leq m_{0}\right)$ vertices already present in the graph. These connections are made by assuming that the probability $\Pi$ that a newly introduced vertex attaches to an existing vertex $i$ is proportional to the connectivity $k_{i}$ of that vertex. Specifically,

$$
\Pi\left(k_{i}\right)=k_{i} / \sum_{\forall j \in V} k_{j}
$$

This is referred to as Preferential Attachment (PA). After $t$ time steps, the graph consists of $t+m_{0}$ vertices and $m t$ edges and possesses a power law distribution of vertex connectivity $P(k)=k^{-}$ $\gamma$, with $\gamma \approx 3$ (Figure 1). Note that so long as $m=m_{0}$, connectivity is guaranteed as the node added at $t=1$ is forced to connect to all of the $m_{0}$ original nodes. 


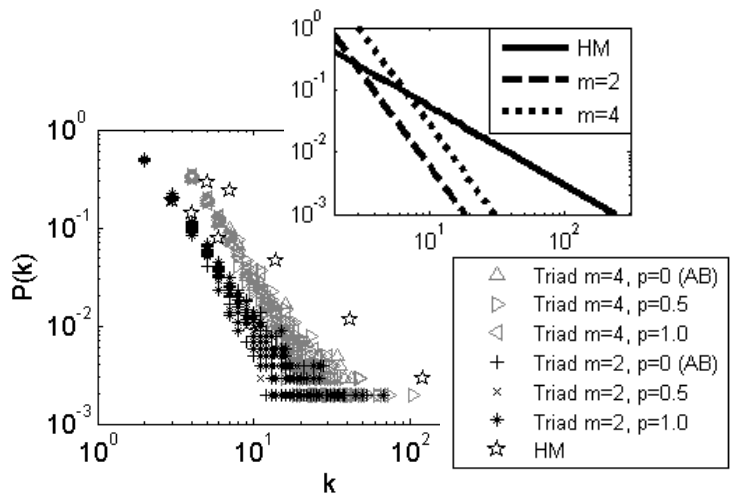

Figure 1. Distributions of vertex connectivity $\mathbf{P}(k)$ for the scale-free graphs considered in this study.

\subsubsection{The Triad Algorithm}

While the $\mathrm{AB}$ algorithm produces graphs with distributions of vertex connectivity that are in good agreement with various realworld data sets [1][3], it has the feature that the clustering coefficient, a quantification of the degree of cliquishness (see section 2.3), decreases with $\mid \mathrm{IV}^{-0.75}$ and asymptotically approaches 0 [26]. Therefore the $\mathrm{AB}$ algorithm does not capture the high clustering found in many instances of real-world scale-free topologies (e.g. email networks [7]). To compensate for this, Holme and Kim [13] proposed a "Triad" algorithm that produces scale-free graphs with power-law distributions of vertex connectivity and high clustering coefficients, such that the clustering coefficient of the generated graph can be tuned using a single parameter.

The Triad algorithm follows the $\mathrm{AB}$ algorithm very closely, but adds an additional step, referred to as Triad Formation (TF), in order to incorporate the high clustering. The Triad algorithm begins with an initial set of $m_{0}$ vertices that are not connected by any edges. At each time step $t$ a new vertex is added to the graph, such that it attaches to $m\left(\leq m_{0}\right)$ vertices already present in the graph. The first of the $m$ connections always occurs according to a PA step (Figure 2a). The rest of the $m$ connections for this newly added vertex use a TF step with probability $p$ and a PA step with probability $(1-p)$. The TF step is defined as follows. If an edge between vertex $i$ and vertex $j$ was added in the previous PA step, then an additional edge from $i$ to a randomly chosen neighbor of $j$
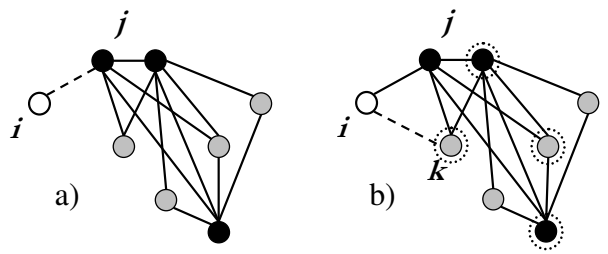

Figure 2. Formation of a Triad graph with $m_{0}=4$ vertices (gray) at time step $t=4$. (a) PA: Node $i$ has just attached to node $j$ (dashed line) where $j$ was selected from all existing nodes with probability proportional to its degree, and (b) TF: node $i$ forms a triad by connecting to node $k$, randomly chosen from all of the neighbors of node $j$ (dashed circles). is added (Figure 2b). If all neighbors of $j$ are already neighbors of $i$ or if $j$ has no neighbors, then a PA step is performed instead.

Like the $\mathrm{AB}$ algorithm, the Triad algorithm produces a graph consisting of $t+m_{0}$ vertices and $m t$ edges at the end of $t$ time steps and possesses a power law distribution of vertex connectivity $P(k)$ $=k^{-\gamma}$, with $\gamma \approx 3$ (Figure 1). This distribution of vertex connectivity is approximately the same for any combination of parameter settings in the $\mathrm{AB}$ or Triad algorithms. However, the absolute distribution of vertex connectivities generated using larger $m$ are shifted to the right relative to those generated using smaller $m$ (Figure 1, inset), since higher $m$ means greater connectivity. In the Triad algorithm, the clustering coefficient can be increased by simply increasing the tunable parameter $p$; note that the Triad algorithm reduces to the $\mathrm{AB}$ algorithm when $p=0$.

\subsubsection{The Hierarchical-Modular Algorithm}

Ravasz et al. [26] demonstrated that the metabolic networks of 43 organisms were not only scale-free, but also displayed exceptionally high clustering. They proposed that this was due to the high modularity of metabolic networks, where densely connected functional modules of various sizes are sparsely attached to other functional subunits in a hierarchical manner. While the Triad algorithm produces scale-free graphs with high clustering, the resulting graphs are not modular or hierarchical. In order to generate scale-free graphs that possess high clustering, modularity, and hierarchical organization, Ravasz et al. [26] proposed a new algorithm, which we refer to as the HierarchicalModular (HM) algorithm.

The algorithm works as follows. In the first iteration (Figure 3a), the algorithm begins with a single, fully connected four-node module. In the second iteration (Figure 3b), three replicas of the module generated in the previous iteration (black) are made. The central nodes of the replicas (gray) are then connected to one another, and each of the peripheral nodes of the replicas (white) are attached to the central node of the original module. This process can be repeated indefinitely, with the size of the graph quadrupling at every iteration.

The graph generated by the HM algorithm possesses a distribution of vertex connectivity $P(k)=k^{-\gamma}$, with $\gamma \approx \ln (4) / \ln (3)$ (Figure 1 ) and a clustering coefficient that converges to an asymptotic limit of $C \approx 0.606$ for reasonably sized networks (i.e. $|V| \approx 1500$ ) [26]. After $t$ time steps, the graph consists of $4^{t}$ vertices and the number a)

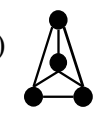

b)

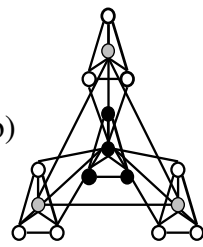

Figure 3. Schematic diagram of the generation of the Hierarchical-Modular (HM) scale-free topology starting from (a) an initial module of 4 fully connected nodes at $t=1$ and proceeding to a topology consisting of (b) 16 nodes at $t=2$. At each time step $t>1$, the central nodes of the generated replicas (grey) connect to one another, peripheral nodes (white) connect to the central node of the original module, and internal nodes, including all of the nodes in the previously existing module (black), do not gain any new connections. 
of edges at time step $t\left(|\mathrm{E}|_{t}\right)$ is given by the recursion:

$$
|E|_{t}=4|E|_{t-1}+3^{t}+3
$$

where $\mid \mathrm{El}_{1}=6$, since the initial four-node module is fully connected.

\subsection{Structural metrics of graphs}

When quantifying the structural properties of a graph, there are several metrics of potential interest. In this section, we briefly define the metrics employed in the current study. The distribution of vertex connectivity, $P(k)$, is a probability distribution function that depicts the frequency with which a given vertex has $k$ connections. The distance $\operatorname{dist}(i, j)$ between two vertices $i$ and $j$ is defined as the length of the shortest path between $i$ and $j$. The diameter $D$ of a graph is defined as the maximum of the distances between all pairs of vertices:

$$
D=\max \{\operatorname{dist}(i, j) \mid i, j \in V\}
$$

The characteristic path length $L_{i}$ of a vertex $i$ is defined as the mean of the shortest paths between vertex $i$ and all other vertices in the graph:

$$
L_{i}=\frac{1}{(|V|-1)} \sum_{\forall j \neq i \in V} \operatorname{dist}(i, j)
$$

The characteristic path length $L$ of a graph $G$ is defined as the mean of the characteristic path lengths of the individual vertices,

$$
L=\frac{1}{|V|} \sum_{\forall i \in V} L_{i}
$$

with standard deviation $\sigma_{L}$. We define the characteristic maximum path length $L_{\max }$ of a graph $G$ as the maximum of the characteristic path lengths,

$$
L_{\max }=\max \left\{L_{i} \mid i \in V\right\}
$$

Finally, the clustering coefficient $C_{i}$ of a vertex $i$ connected to $k_{i}$ nodes is defined as the ratio between the number of edges, $E_{i}$, that actually exist between the $k_{i}$ nodes and the number of edges that could potentially exist between the $k_{i}$ nodes:

$$
C_{i}=\frac{2 E_{i}}{k_{i}\left(k_{i}-1\right)}
$$

and the clustering coefficient of a graph $G$ is given by:

$$
C=\frac{\sum_{\forall i \in V} C_{i}}{|V|}
$$

with standard deviation $\sigma_{C}$.

\subsection{Takeover time}

Consider a population with only two levels of fitness; i.e. let $\Lambda_{i}(t)$ be the fitness value of vertex $i \in V$ at time $t$, where $\Lambda_{i}(t) \in\{0,1\}$ and 1 is more fit than 0 . In the initial population, $\Lambda_{i}(0)=1$ for exactly one $i \in V$ and $\Lambda_{j}(0)=0 \forall j \neq i \in V$. Let $N_{t}$ denote the number of nodes with value 1 at time $t$ :

$$
N_{t}=\sum_{\forall i \in V} \Lambda_{i}(t)
$$

Following [27], we define the takeover time $T=\min \left\{t\left|N_{t}=\right| V \mid\right\}$ to be the minimum number of generations such that the most fit individual fully saturates the entire population. The simulations performed herein are nonextinctive [27], such that $N_{t}$ can never decrease. $\hat{E}_{i}[\mathrm{~T}]$ is defined as the empirical estimate of the expected takeover time given that the initial best individual is located in vertex $i$. Thus, the overall empirically estimated expected takeover time is simply

$$
\widehat{E}[T]=\frac{1}{|V|} \sum_{\forall i \in V} \widehat{E}_{i}[T]
$$

assuming that the initial best individual is equally likely to appear in any given node.

\subsection{Selection}

In order to most directly infer the influence of population structure on takeover time dynamics, selection was implemented using a simple "replace if better" strategy, where nodes were updated synchronously, as follows. For each node $i \in V$, a node $j$ was selected at random with uniform probability from the mating neighborhood of node $i$, with neighborhood size of size $k_{i}$. Thus, if there are $x$ nodes containing the fittest value in the mating neighborhood of node $i$, then the probability of selecting one of them is simply $x / k_{i}$. The value of the selected node $j$ then replaced the value of node $i$ if $\Lambda_{j}(t)>\Lambda_{i}(t)$.

\subsection{Experimental Design}

For all simulations, the population size was held constant at 1024 individuals (i.e. $|V|=1024$ ). We investigated the takeover times of populations structured on several instances of scale-free spatial topologies generated using the $\mathrm{AB}$ algorithm, the Triad algorithm, and the HM algorithm. While analytical solutions or mathematical

\begin{tabular}{|c|c|c|c|c|c|}
\hline \multicolumn{2}{|c|}{ Topology } & $m$ & $p$ & Instances & $\begin{array}{c}\text { Simulations } \\
\text { per vertex }\end{array}$ \\
\hline \multirow{7}{*}{ 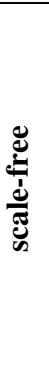 } & $\mathbf{A B}$ & 4 & 0.0 & 10 & 10 \\
\hline & Triad & 4 & 0.5 & 10 & 10 \\
\hline & Triad & 4 & 1.0 & 10 & 10 \\
\hline & $\mathbf{A B}$ & 2 & 0.0 & 10 & 10 \\
\hline & Triad & 2 & 0.5 & 10 & 10 \\
\hline & Triad & 2 & 1.0 & 10 & 10 \\
\hline & $\mathbf{H M}$ & - & - & 1 & 100 \\
\hline \multicolumn{2}{|c|}{ Panmictic } & - & - & 1 & 100 \\
\hline \multicolumn{2}{|c|}{ 2D Lattice } & - & - & 1 & 100 \\
\hline
\end{tabular}
approximations of expected takeover times are possible for vertextransitive graphs such as 1D and 2D toroidal lattices [10][27], the extreme heterogeneity of scale-free topologies makes such mathematical analysis impractical [21]. We therefore performed an empirical analysis, deriving expected takeover times through rigorous simulation. As a baseline for comparison, we also

Table 1. Summary of the parameter settings used for each spatial topology considered in this study. 
Table 2. Structural characteristics of the topologies considered in this study. For stochastically generated graphs (i.e. AB and Triad), the data represents the average over all instances.

\begin{tabular}{|c|c|c|c|c|c|c|c|c|c|}
\hline \multicolumn{2}{|c|}{ Topology } & $m$ & $p$ & $D$ & $L$ & $\sigma_{L}$ & $L_{\max }$ & $C$ & $\sigma_{C}$ \\
\hline \multirow{7}{*}{$\frac{\mathbb{E}}{\frac{d}{d}}$} & $\mathbf{A B}$ & 4 & 0.0 & 5 & 3.19 & 0.26 & 3.80 & 0.03 & 0.07 \\
\hline & Triad & 4 & 0.5 & 5.2 & 3.26 & 0.28 & 4.07 & 0.24 & 0.15 \\
\hline & Triad & 4 & 1.0 & 7 & 3.58 & 0.43 & 5.11 & 0.54 & 0.21 \\
\hline & $\mathbf{A B}$ & 2 & 0.0 & 7.3 & 4.15 & 0.43 & 5.42 & 0.02 & 0.11 \\
\hline & Triad & 2 & 0.5 & 8.5 & 4.32 & 0.50 & 6.08 & 0.39 & 0.40 \\
\hline & Triad & 2 & 1.0 & 10.7 & 4.88 & 0.80 & 7.61 & 0.74 & 0.29 \\
\hline & HM & - & - & 9 & 3.97 & 0.87 & 5.89 & 0.63 & 0.25 \\
\hline \multicolumn{2}{|c|}{ Panmictic } & - & - & 1 & 1 & 0 & 1 & 1 & 0 \\
\hline \multicolumn{2}{|c|}{ 2D Lattice } & - & - & 16 & 10.68 & 0 & 10.68 & 0.40 & 0 \\
\hline
\end{tabular}

investigated takeover times in panmictic population structures and square $32 \times 322$ D toroidal lattice population structures with $3 \times 3$ Moore neighborhoods of interactions. The parameter settings used in each graph-generating algorithm are provided in Table 1, with resulting topological metrics as shown in Table 2. For all graphs generated using the $\mathrm{AB}$ and Triad algorithms, $m_{0}=m$.

Since the expected takeover time $(\hat{E}[T])$ in scale-free population structures is highly dependent upon the placement of the initial copy of the best individual, we systematically placed the best individual of the initial population in each node of the topology one at a time, and subsequently performed ten independent takeover time simulations for each individual placement, in order to mitigate the stochasticity in the selection policy. Further, since the $\mathrm{AB}$ and Triad graph-generating algorithms are themselves stochastic in nature, we also created 10 instances of each of these types of graphs for each combination of parameter settings (Table 1). In order to have the same number of trials per graph type, we ran one hundred takeover time simulations for each initial vertex containing the best individual in the deterministically created graphs (panmictic population structures, two-dimensional toroidal lattices, and the scale-free topologies generated using the HM algorithm) (Table 1), resulting in a total of 102,400 independent takeover time simulations for each type of graph considered.

\section{EXPERIMENTAL RESULTS}

Figure 4 depicts the observed takeover time dynamics of populations structured on scale-free spatial topologies generated using the $\mathrm{AB}(p=0)$ and Triad $(\mathrm{p} \in\{0.5,1.0\})$ algorithms with $m=$ 4 (Figure $4 \mathrm{a}$ ) and $m=2$ (Figure $4 \mathrm{~b}$ ), and using the HM algorithm (Figure 4c). Specifically, at each generation $t$ we plot number of nodes containing the maximum fitness $\left(N_{t}\right)$, averaged over all

Figure 4. Observed takeover time dynamics for populations structured on scale-free topologies generated using (a) the AB and Triad algorithms with $m=4$, (b) the AB and Triad algorithms with $m=2$, and (c) the HM algorithm. For comparison, each graph also shows the takeover time dynamics for panmictic populations (open circles) and populations structured on a $32 \times 32$ toroidal lattice (filled squares).
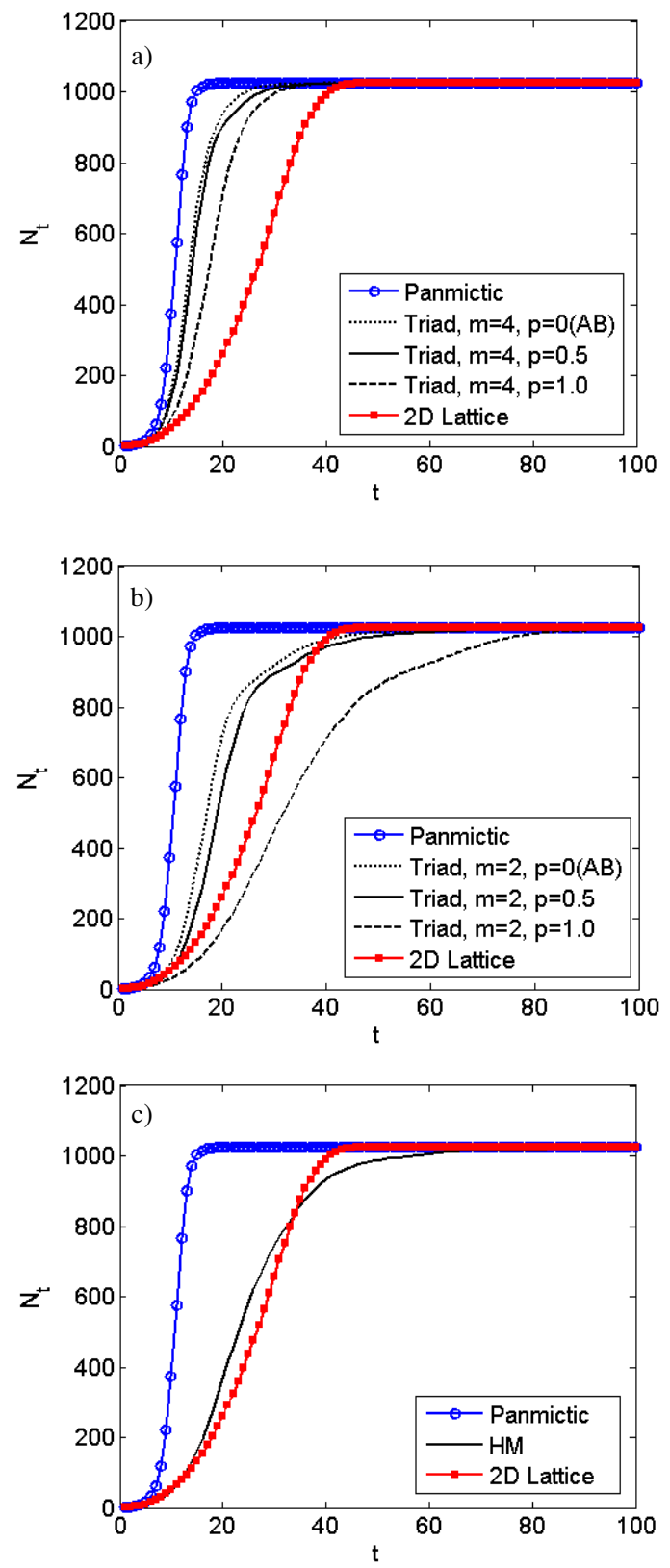
Table 3. Empirically estimated mean, standard deviation, minimum, and maximum takeover time for each spatial topology considered in this study.

Takeover time

\begin{tabular}{|c|c|c|c|c|c|c|c|}
\hline \multirow{2}{*}{\multicolumn{2}{|c|}{ Topology }} & \multirow[b]{2}{*}{$m$} & \multirow[b]{2}{*}{$p$} & \multirow[b]{2}{*}{ mean } & \multirow{2}{*}{\multicolumn{2}{|c|}{ std $\quad \min$}} & \multirow{3}{*}{$\begin{array}{r}\max \\
66\end{array}$} \\
\hline & & & & & & & \\
\hline \multirow{7}{*}{ 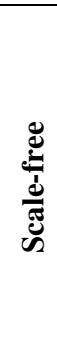 } & $\overline{A B}$ & 4 & 0.0 & 19.16 & 3.39 & 12 & \\
\hline & Triad & 4 & 0.5 & 20.16 & 3.48 & 12 & 60 \\
\hline & Triad & 4 & 1.0 & 26.36 & 4.19 & 15 & 55 \\
\hline & $\mathbf{A B}$ & 2 & 0.0 & 25.26 & 5.38 & 14 & 89 \\
\hline & Triad & 2 & 0.5 & 29.79 & 6.55 & 17 & 146 \\
\hline & Triad & 2 & 1.0 & 60.57 & 14.43 & 23 & 208 \\
\hline & $\mathbf{H M}$ & - & - & 37.34 & 15.51 & 13 & 351 \\
\hline \multicolumn{2}{|c|}{ Panmictic } & - & - & 14.83 & 1.35 & 11 & 26 \\
\hline \multicolumn{2}{|c|}{ 2D Lattice } & - & - & 44.15 & 2.26 & 35 & 57 \\
\hline
\end{tabular}

102,400 trials on that graph type. As a baseline for comparison, the observed takeover time dynamics of populations structured on panmictic topologies and 2D toroidal lattice topologies with $3 \times 3$ Moore neighborhoods are also provided. Table 3 provides a summary of the observed takeover times. All growth curves for takeover of the best individual are sigmoidal, exhibiting exponential growth followed by saturation (Figure 4). In all cases, the selective pressures induced by the spatially-structured topologies are less than that of the panmictic topology (Figure 4). However, the relationship between the selective pressures induced by the scale free topologies relative to that induced by a 2D lattice varies, as discussed below.

\subsection{Albert-Barabàsi and Triad scale-free topologies, $\mathrm{m}=4$}

When $m=4$, the selective pressures induced by the scale-free topologies are consistently greater than that of the 2D lattice topology (Figure 4a). As $p$ increases from $0(\mathrm{AB})$ to 0.5 to 1 (Triad), the selective pressure decreases, with a more noticeable decrease occurring between $p=0.5$ and $p=1.0$ than between $p=$ 0 and $p=0.5$. While the maximum takeover times were similar to those on the 2D lattice (Table 3, column 7), the minimum takeover times were closer to those on the panmictic topology (Table 3, column 6), and thus the average takeover times on these scale free graphs were less than on the 2D lattice (Table 3, column 4).

\subsection{Albert-Barabàsi and Triad scale-free topologies, $\mathbf{m}=\mathbf{2}$}

When $m=2$, the period of saturation in the scale free topologies begins earlier and lasts longer than the period of saturation in either the lattice or panmictic topologies, such that the growth curves for scale-free topologies with $p=0$ and $p=0.5$ ultimately cross under the curve for the 2D lattice (Figure $4 \mathrm{~b}$ ), due to the fact that the maximum takeover times were much higher than on the 2D lattice (Table 3, column 7). Thus, for $p=0$ and $p=0.5$, the selective pressure induced by these topologies is stronger than that of the lattice topology in early generations, but weaker in latter generations. However, the average takeover times for these scale free graphs are still much lower than on the 2D lattice (Table 3 , column 4). In contrast, the selective pressure induced by scalefree topologies generated using the Triad algorithm with $p=1$ is consistently weaker than the selective pressure induced by the $2 \mathrm{D}$ lattice topology throughout the entire evolution of the population. Indeed, the empirically estimated takeover time $(\hat{E}[T])$ of populations evolving on scale-free topologies generated using the Triad algorithm with $p=1$ is nearly 1.5 times that of populations evolving on the 2D lattice (Table 2, column 4), due to the much greater maximum (Table 3, column 7), and consequently much higher standard deviation (Table 3 , column 5), of the takeover times on the Triad graph with $p=1$, relative to those of the $2 \mathrm{D}$ lattice.

\subsection{The Hierarchical-Modular topology}

While the shape of the growth curve of populations structured on the HM scale-free topology is still sigmoidal, the period of saturation begins even earlier than the period of saturation in the $\mathrm{AB}$ and Triad scale-free topologies (compare Figure $4 \mathrm{c}$ with Figures $4 \mathrm{a}$ and $4 \mathrm{~b}$ ). Similar to the growth curves observed on topologies generated using the $\mathrm{AB}(m=2)$ and Triad $(m=2, p=$ $0.5)$ algorithms, the selective pressure induced by HM scale-free topologies is stronger than that induced by the lattice topology in early generations, but weaker in latter generations. Surprisingly, although the maximum takeover time is highest in the HM graph (Table 3, column 7), its average takeover time remains below that of both the 2D lattice and the Triad with $m=2$ and $p=1.0$, presumably because the minimum takeover time is so low for HM (Table 3, column 6).

\subsection{Relationship between topology and mean takeover time}

The characteristic path length $(L)$ of a spatial topology plays a large role in governing the rate at which advantageous genetic information can propagate throughout a population. Note that in Figures $5 \mathrm{a}$ and $5 \mathrm{~b}$, we include data for $2 \mathrm{D}$ lattices with the $3 \times 3$ neighborhoods, but also for larger square neighborhoods with diameters $d \in\{5,7,9,11\}$. Figure 5a depicts a strong linear correlation $\left(\mathrm{R}^{2}=0.88\right)$ between $L$ and the minimum observed takeover time $(\min (\hat{E}[T]))$ on each spatial topology considered in this study. However, the correlation between $L$ and the mean observed takeover time $(\hat{E}[T])$ is considerably weaker $\left(\mathrm{R}^{2}=0.38\right)$, suggesting that other topological properties must also play an important role. Consequently, we searched for statistical correlations between the various topological metrics shown in Table 2 (both individually and in various combinations) and the mean takeover times shown in Table 3, with the result that the strongest correlation incorporates both $\sigma_{L}^{2}$, and $L_{\max }$, as discussed below.

Non-regular topologies, such as the scale-free population structures considered here, have a large variance in their structural characteristics, while regular topologies, by definition, have zero variance (Table 2$)$. Since the mean takeover time $(\hat{E}[T])$ is the average of all of the takeover times starting with the initial copy of the best individual in every node (eq. 10), the variability in the structural properties of a non-regular spatial topology must also 
be taken into account in order to more accurately infer the expected takeover time. We found that the relation,

$$
\widehat{E}[T] \propto L_{\max }+\sigma_{L}^{2} L_{\max }
$$

has a strong correlation $\left(\mathrm{R}^{2}=0.91\right.$, Figure $\left.5 \mathrm{~b}\right)$. Note that for regular topologies this relationship (11) still holds, but the second term drops out since $\sigma_{L}^{2}=0$ and $\hat{E}[T]$ is simply a linear function of only $L_{\max }$. It is worth noting that a structural analysis of the AB scale-free topology generated using the parameters in [9] $(\mathrm{IVI}=$ 1024, $m_{0}=14, m=10$ ) predicts an expected takeover time that is close to that of a panmictic population structure, consistent with the results presented in [9] (data not shown).

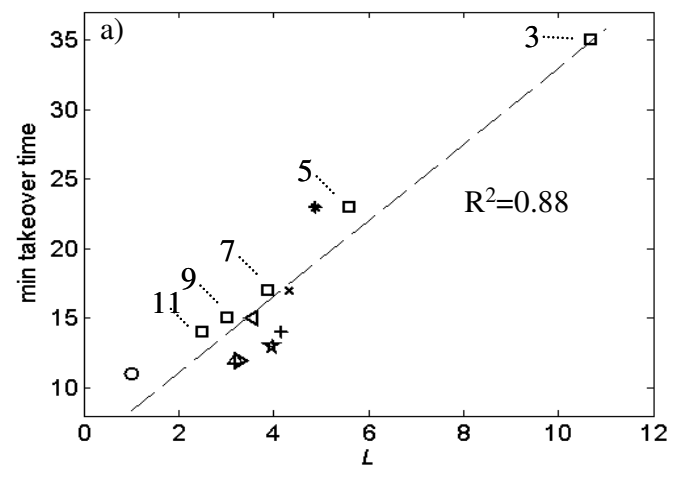

\begin{tabular}{|ll|}
$\Delta$ & Triad $m=4, p=0(\mathrm{AB})$ \\
$\mathrm{p}$ & Triad $m=4, p=0.5$ \\
1 & Triad $m=4, p=1.0$ \\
+ & Triad $m=2, p=0(\mathrm{AB})$ \\
$\times$ & Triad $m=2, p=0.5$ \\
$*$ & Triad $m=2, p=1.0$ \\
$*$ & HM \\
0 & Panmictic \\
$\square$ & 20 Lattice \\
\hline
\end{tabular}

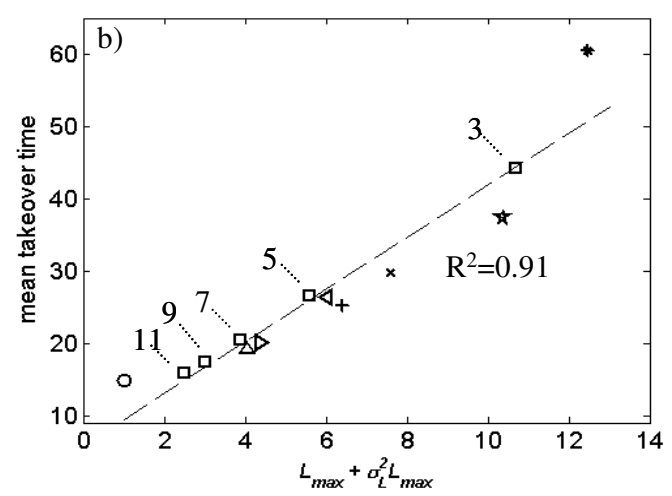

Figure 5. a) Observed minimum takeover time as a linear function of the characteristic path length $(L)$ of the spatial topology. b) Observed mean takeover time as a linear function of $L_{\max }+\sigma_{L}^{2} L_{\text {max }}$. For the 2D lattices (open squares), the diameter $\boldsymbol{d} \in\{3,5,7,9,11\}$ of the Moore neighborhood is indicated.

\section{DISCUSSION AND CONCLUSIONS}

In [9], takeover time dynamics were investigated on one particular instance of a scale-free topology generated using the $\mathrm{AB}$ algorithm $\left(|V|=1024, m_{0}=14, m=10\right)$. They concluded that the takeover times of populations on scale-free topologies were at least as fast as the takeover times of populations using a random or panmictic topology. Here, we show that the selective pressure induced by a scale-free population structure is actually highly dependent upon its additional topological properties and can be tuned from a selective pressure nearly as strong as that induced by a panmictic topology to a selective pressure that is even weaker than that induced by a square, 2D lattice with $3 \times 3$ Moore neighborhoods.

A structural analysis of the spatial topologies considered in this study revealed a strong linear correlation between the characteristic path length $(L)$ of the population structure and the minimum observed takeover time $(\min (\hat{E}[T]))$. Since $L$ is the mean shortest path between all pairs of vertices, this relationship makes intuitive sense; the fastest propagation of the best individual most likely occurred along at least a subset of these shortest paths. However, the correlation between $L$ and the mean observed takeover time $(\hat{E}[T])$ was shown to be very weak, resulting from the extreme heterogeneity of the scale-free topologies considered in this study, which have high variance in their structural characteristics. Our empirical observations across all topologies considered suggest that the mean expected takeover time can be inferred with reasonable accuracy as a function of only $L_{\max }$ and $\sigma_{L}^{2}(11)$. It is interesting to note that replacing $L_{\max }$ with $L$ in relation (11) results in a weaker correlation $\left(\mathrm{R}^{2}=0.75\right)$. Since the vertex associated with $L_{\max }$ must always be reached for complete takeover to occur, the mean takeover time is more directly correlated with this metric than with $L$, which captures the average of all shortest paths. To account for the heterogeneity in the topologies considered, we found that $\sigma^{2}$ plays a governing role, capturing the variability in the shortest paths present in the population structure. Using such a structural analysis to estimate mean takeover time may prove useful, as only a few simple calculations regarding the underlying topology are required, precluding the need to run computationally expensive simulations, as performed herein, or recursive probabilistic formulations (e.g., [31]).

Gaining a better understanding of how the topological properties of population structure affect information flow through networks (selective pressure in evolving systems) can also provide useful insights into the behaviors of both natural and artificial systems. As the important influence of the spatial constraints on interindividual interactions has become increasingly appreciated, spatially-explicit models of evolutionary dynamics have tended to employ more biologically meaningful population structures. Several studies have shown that limiting the spatial scale of interaction events facilitates the emergence of evolutionary phenomena that would be otherwise impossible in a globally interacting population. For example, the spatial locality of interindividual interactions has been shown to have an important influence on the maintenance of genetic diversity [15][29], the evolution of altruism [33], and the suppression of evolutionary pathologies [2]. Topological analyses of interaction networks have proven useful in understanding the sensitivity of selforganized speciation [24] and predator-prey dynamics [25] to 
occasional long-distance interaction events. The empirical relationship derived here may offer an easy means of rapidly estimating the dynamics of information flow on disparate population structures, which may prove useful in a variety of diverse applications, such as estimating the impact of implementing evolutionary algorithms on various topologies or understanding the impact of social network topologies on the spread of ideas or disease. This approach can also provide useful insight into the topological properties that most directly affect network dynamics.

Future work will expand upon the results presented in this study by investigating the relationship between the topological properties of population structure and takeover time dynamics on a wider class of heterogeneous interaction topologies and using alternative selection methods.

\section{Acknowledgements}

This work was supported in part by a graduate research assistantship and a Pilot Award funded by DOE-FG0200ER45828 awarded by the US Department of Energy through its EPSCoR program. We thank Dr. Josh Bongard for his helpful comments on an earlier draft of this manuscript.

\section{References}

[1] Albert, R., Jeong, H., \& Barabàsi, A.L. Diameter of the World-Wide Web. Nature, 401 (1999), 130-131.

[2] Altenberg, L. Evolvability suppression to stabilize far-sighted adaptations. Artificial Life, 11, 4 (2005), 427-443.

[3] Barabàsi, A.L. \& Albert, R. Emergence of scaling in random networks. Science, 286 (1999), 509-512.

[4] Barabàsi, A.L., Ravasz, E., \& Vicsek, T. Deterministic scale-free networks. Physica A, 299 (2001), 559-564.

[5] Caldarelli, G., Capocci, A. De Los Rios, P., \& Muñoz, M.A. Scalefree networks from varying vertex intrinsic fitness. Physical Review Letters, 89, 25 (2002), 258702.

[6] Callaway, D.S., Newman, M.E.J., Strogatz, S.H., \& Watts, D.J. Network robustness and fragility: percolation on random graphs. Physical Review Letters, 85, 25 (2000), 5468.

[7] Ebel, H., Mielsch, L., \& Bornholdt, S. Scale-free topology of e-mail networks. Physical Review E, 66 (2002), 035103(R).

[8] Falconer, D.S. \& Mackay, T.F.C. Quantitative Genetics. Pearson Education Limited, London, UK, 1996.

[9] Giacobini, M., Tomassini, M., \& Tettamanzi, A. Takeover time curves in random and small-world structured populations. In Proc. Genetic and Evolutionary Computation Conference. ACM Press, New York, NY, 2005, 1333-1340.

[10] Giacobini, M., Tomassini, M., Tettamanzi, A., \& Alba, E. Selection intensity in cellular evolutionary algorithms for regular lattices. IEEE Transactions on Evolutionary Computation, 9, 5 (2005), 489505.

[11] Goldberg, D.E., \& Deb, K. A comparative analysis of selection schemes used in genetic algorithms. In Proc. Foundations of Genetic Algorithms. Morgan-Kauffman, San Mateo, CA, 1991, 6993.
[12] Holland, J. Adaptation in Natural and Artificial Systems. MIT Press, Cambridge, MA, 1992.

[13] Holme, P. \& Kim, B.J. Growing scale-free networks with tunable clustering. Physical Review E, 65 (2002), 026107.

[14] Jeong, H., Mason, S.P., Barabàsi, A.L., \& Oltvai, Z.N. Lethality and centrality in protein networks. Nature, 411 (2001), 41-42.

[15] Kerr, B., Riley, M.A., Feldman, M.W., \& Bohannan, B.J.M. Local dispersal promotes biodiversity in a real life game of rock-paperscissors. Nature, 418 (2002), 171-174.

[16] Klemm, K. \& Eguíluz, V.M. Highly clustered scale-free networks. Physical Review E, 65 (2002), 036123

[17] Liljeros, F., Edling, C.R., Amaral, L.A.N., Stanely, H.E., \& Åberg, Y. The web of human sexual contacts. Nature, 411 (2001), 907-908

[18] Motter, A.E., de Moura, A.P.S., Lai, Y.C., \& Dasgupta, P. Topology of the conceptual network of language. Physical Review E, 65 (2002), 065102(R).

[19] Nathan, R. Long-distance dispersal of plants. Science, 313 (2006), 786-788.

[20] Newman, M.E.J. The structure and function of complex networks. SIAM Review, 45 (2003), 167-256.

[21] Ohtsuki, H., Hauert, C., Lieberman, E., \& Nowak, M.A. A simple rule for the evolution of cooperation on graphs and social networks. Nature, 441, 25 (2006), 502-505.

[22] Park, K., Lai, Y.C., \& Ye, N. Self-organized scale-free networks. Physical Review E, 72 (2005), 026131.

[23] Pastor-Satorras, R. \& Vespignani, A. Epidemic spreading in scalefree networks. Physical Review Letters, 86, 14 (2001), 3200.

[24] Payne, J.L., Eppstein, M.J., \& Goodnight, C.J. Sensitivity of selforganized speciation to long-distance dispersal. In Proceedings of the IEEE Symposium on Artificial Life, (2007), 1-7.

[25] Rauch, E.M. \& Bar-Yam, Y. Long-range interactions and evolutionary stability in predator-prey systems. Physical Review E, 73 (2006), 020903.

[26] Ravasz, E., Somera, A.L., Mongru, D.A., Oltvai, Z.N., \& Barabàsi, A.L. Hierarchical organization of modularity in metabolic networks. Science, 297 (2002), 1551-1555.

[27] Rudolph, G. On takeover times in spatially structured populations: array and ring. In Proc. $2^{\text {nd }}$ Asia-Pacific Conference on Genetic Algorithms and Applications. Global-Link Publishing Company, Hong Kong, 2000, 144-151.

[28] Sarma, J. \& De Jong, K. An analysis of the effect of the neighborhood size and shape on local selection algorithms. In Proc. Parallel Problem Solving from Nature Conference. SpringerVerlag, Heidelberg, 1996, 236-244.

[29] Sayama, H., Kaufman, L., \& Bar-Yam, Y. Spontaneous pattern formation and genetic diversity in habitats with irregular geographical features. Conservation Biology, 17 (2003), 893-900.

[30] Servedio, V.D.P., Caldarelli, G., \& Buttà, P. Vertex instrinsic fitness: how to produce arbitrary scale-free networks. Physical Review E, 70 (2004), 056126

[31] Sprave, J. A Unified model of non-panmictic population structures in evolutionary algorithms. In Proc. of the Congress of Evolutionary Computation Conference. IEEE Press, 1999, 1384-1391.

[32] Siganos, G., Faloutsos, M., Faloutsos, P., \& Faloutsos, C. Power laws and the AS-level internet topology. IEE/ACM Transactions on Networking, 11, 4 (2003), 514-524.

[33] Werfel, J. \& Bar-Yam, Y. The evolution of reproductive restraint through social communication. PNAS, 101, 30 (2004), 1101911024. 